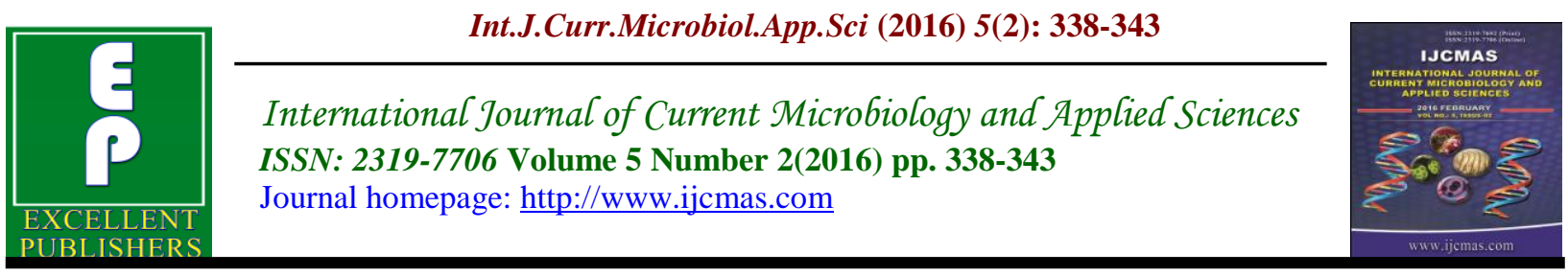

Original Research Article

doi: http://dx.doi.org/10.20546/ijcmas.2016.502.039

\title{
Sensitivity and specificity of Anti-mutated Citrullinated vimentin antibodies compared with Anti-cyclic Citrullinated Peptides in patients of Rheumatoid arthritis
}

\author{
Saurabh Agarwal*, Sippy Agrawal, Manoj Kumar and Arun Kumar Pandey \\ Institution M.L.B. Medical College Jhansi (U.P), India \\ *Corresponding author
}

\begin{abstract}
A B S T R A C T
Keywords

Anti-cyclic

citrullinated

peptide

(anti-CCP2);

Anticitrullinated

vimentin

antibody

(anti-CMV);

Rheumatoid

arthritis (RA).

Article Info

Accepted:

17 January 2016

Available Online:

10, February 2016

Rheumatoid arthritis is a chronic systemic inflammatory autoimmune disease primarily characterized by a bilateral symmetrical polyarticular arthritis, which is often erosive.The last 5 years have seen the emergence and establishment of antibodies to citrullinated antigens as the diagnostic marker for rheumatoid arthritis (RA). Initially, these were detected using a synthetic peptide, which has undergone a number of modifications to give a diagnostic test with a sensitivity of $65-80 \%$ and a specificity of $>95 \%$. Antibodies to citrullinated vimentin were first described in 1994 as a highly specific marker for RA (anti-Sa). However, no easily performed assay for these antibodies has been available. Among them, antibodies against cyclic citrullinated peptides (CCP) are useful for diagnosing RA. antibodies to mutated citrullinated vimentin (MCV) were described recently in RA. The aim of this study was to determine the diagnostic values of ACCP compared to anti-MCV in rheumatoid arthritis patients. This study included 92 patients with Rheumatoid arthritis (RA) an 35 matching healthy controls. Blood samples were obtained from patients and controls for erythrocyte sedimentation rate (ESR),C reactive protein (CRP), rheumatoid factor (RF). Anti-CCP2 and anti-MCV were determined using ELISA technique. RA group was significantly higher than control group as regard ESR, CRP, RF, Anti-CCP, and Anti- MCV. It was concluded, compared to ACCP, anti-MCV has approximately the same accuracy for the diagnosis of rheumatoid arthritis.
\end{abstract}

\section{Introduction}

Rheumatoid arthritis is a chronic systemic inflammatory autoimmune disease primarily characterized by a bilateral symmetrical polyarticular arthritis, which is often erosive. It is probably the most common autoimmune disease (about $1 \%$ of the world population), affects three times as many women as men, and usually appears in middle age.
Although the etiology of RA remains unknown, it is widely accepted that multiple accumulative/compounding geneticand environmental'hits' are required between the initiation of self-peptide recognition, subsequent loss of tolerance, and the development of autoimmunity. Disease onset is often insidious, initially affecting the small joints of the hands (proximal 
interphalangeal joints), feet, and wrists. Clinical course of the disease varies between individuals but may be mild, relapsingremitting, or progressive. The systemic complications and the severity of articular manifestations (with associated functional impairment) may have a debilitating and disabling impact on the patient, causing significant morbidity, reduced quality of life, and even premature mortality. It is therefore important that the disease is diagnosed and treated early to slow/stop joint damage, increase/maintain joint function, establish remission, and maximize quality of life.

Considering the aggressive nature of the disease process with the significant residual disability, the International Societies of Rheumatology potentially recommended early aggressive treatment for tight control of the inflammatory process aiming to prevent joint destruction and preserve function. Rheumatoid factors (RFs)were the first biological markers discovered for RA and remain the only laboratory criterion included in the American College of Rheumatology criteria for RA classification (Arnett et al., 1988). The currently laboratory diagnostics of RA particularly early RA, is based on a highly specific marker of the disease such as antibodies against citrullinated proteins. The positive test for anti-cyclic citrullinated protein (ACCP) antibody is now used as a classification criterion of $\mathrm{RA}(3)$.

ACCP positivity predisposes individuals to more advanced course of the disease, with extensive bony erosions, accelerated atherothrombotic disease and worse overall prognosis (5).

Anti-mutated citrullinated vimentin (Anti$\mathrm{MCV})$ is another anti-citrullinated antibody reacting with mutated citrullinated vimentin
(6). Vimentin is an intermediate filament that is widely expressed by mesenchymal cells and macrophages and easy to detect in the synovium. Modification of the protein occurs in macrophages undergoing apoptosis, and antibodies to citrullinated vimentin may emerge if the apoptotic material is inadequately cleared (Vossenaar et al., 2004). Recently, citrullinated vimentin, a protein highly released in synovial microenvironment, has been identified as potential autoantigen in the pathophysiology of RA and an enzymelinked immunosorbent assay (ELISA) for the detection of Antibodies directed against a mutated citrullinated vimentin (anti-MCV) was developed (Bartoloni et al., 2012).

The objective of this work was to investigate the seropositivity to antibodies against modified citrullinated vimentin antibodies (anti-MCV) in comparison with anti-CCP2.

\section{Materials and Methods}

This case control study was conducted on RA patients attending the outpatient clinic of orthopaedics of M.L.B.Medical College and Hospital, in the period between Jan 2012 to September 2013. Ninty two patients (66 females and 26 males) fulfilling the 1987 American College of Rheumatology (ACR) criteria for a diagnosis of RA were studied, thirty five healthy age and sex matched control subjects (23females, 12 males) were included for comparative assessment of the investigated serological disease markers.

At enrollment caretakers provided informed consents and the following data were collected: age, gender, duration of RA, treatment and hospital admission. The RA group Comprised 92 patients of age ranged from 18-60 years. They were 66 females and 26 males. Their disease duration ranged from 6 months to 22 years. Diagnosis of RA 
was based and confirmed according to (ACR)/(EULAR) 2010 criteria.

\section{Methods}

Six $\mathrm{mL}$ of peripheral venous blood were withdrawn aseptically from each patient and from each control subject. Two $\mathrm{mL}$ blood were left to clot for 15 minutes then centrifuged and sera were put into aliquots and stored at $-20^{\circ} \mathrm{C}$ until assayed for antiMCV and anti CCP2 antibodies for both patients and controls. The remaining $3 \mathrm{ml}$ were used for other investigations done to patients:

a) Complete blood picture $\mathrm{CBC}$ was performed on The CELL-DYN 3700 automated hematology analyzer.

b) Renal and liver function tests were performed on Autoanalyzer Bechman Synchron cx 5 system.

c) Measurement of ESR by the Westergren method.

d) Serum CRP concentrations were determined by immuno-nephelometry methods on a Turbox nephelometer (Orion Diagnostica, Finland). Thetiter of $6 \mathrm{mg} / \mathrm{l}$ were considered positive for CRP.

e) Rheumatoid factor IgM isotype was analyzed using the ELISA kit for RF IgM quantitation (Orgentec Diagnostika $\mathrm{GmbH}$, Germany) according to the manufacturer's instructions. The titer of $20 \mathrm{IU} / \mathrm{ml}$ was regarded as positive(18).

Anti MCV and Anti CCP testing was performed in an investigator blinded fashion. Anti CCP antibody reactivity was tested using a commerciallyavailable automated ELISA on automated analyser according to manufacturer recommendation. Value of $25.0 \mathrm{U} / \mathrm{ml}$ or greater were considered to be positive.

Anti MCV antibodies were measured using a commercially available ELISA according to manufacturer instruction. Value of $20.0 \mathrm{U} / \mathrm{ml}$ or greater considered to be positive.

Serum diluted 1: 1000 for anti mcv and 1:50 for anti CCP. Than incubated on coated plates with kit standard and control at room temperature for $30 \mathrm{~min}$. than washed and added horseradish peroxidase labelled anti human IgG for $15 \mathrm{~min}$. the reaction were revealed by the addition of TBM $(3,3 ; 5,5-$ tetramethylbenzidine) substrate and the colour intensity was measured at 450/620 $\mathrm{nm}$.

\section{Results and Discussion}

Laboratory and serological assessment showed a mean serum Anti-CCP2 in patients with RA which was significantly higher than controls. The anti-MCV levels were also significantly higher in RA patients compared to healthy control subjects.

Of 92 patients with RA, 72 patients were positive for anti-MCV antibodies (78.26\%), 70 patients were positive for anti-CCP antibodies $(76.08 \%), 54$ patients were positive for RF $(58.60 \%)$, any of them were positive in 89 patients $(96.73 \%)$ and all of them positive in 34 patients (37\%). By contrast, of 35 healthycontrols, 1 person was positive for anti-MCVantibodies (97.14\%), 2 persons were positive for anti-CCP antibodies (94.28\%), 4 persons were positive for RF $(77.15 \%)$ any of them were positive in 11 subjects (68.57\%)and all of them positive in no subjects $(0 \%)$.

The main focus of our study was to 
investigate the usefulness of anti-MCV for diagnosing and assessing severity of RA in comparison to anti-CCP. In recent years, many studies have evaluated the presence of anti-MCV, anti-CCP antibodies, in RA patients. In our study, at the cutoff values recommended by the manufacturer, the sensitivity and specificity of anti-CCP, and anti-MCV in diagnosing RA compared.

In most of the published works that we studied, the sensitivity of anti-MCV was somehow higher than ACCP but ACCP was more specific $(7,9-11,14)$. The same results have been mentioned in some other studies that Ernest Wagner et al. referred to. They found that in RF negative patients, the sensitivity of anti-MCV is higher $(43.8 \%$ versus $30 \%$ ) (11).

The study showed that the levels of anti$\mathrm{CCP} 2$ were significantly increased in the sera of patients with RA in comparison with the controls, which agrees with what has been reported in late studies by Zhu and Feng, 2013 in chinese patients with RA, Sariyildiz et al. 2013 in Turkish patients amongst other multi-ethenic studies (15-18). In confirmation to what has been previously reported the study revealed a significantly higher serum anti-MCV antibody level in Egyptian RA patients when compared to healthy controls, supporting the hypothesis that citrullinated vimentin plays an integral role in triggering the inflammatory immune response in RA $(18,19)$. This antigenic selfprotein activates $\mathrm{T}$ lymphocytes by binding on HLA-DR4 on the surface of antigen presenting cells and may contribute to certain pathways in the pathogenesis of RA. Several late studies have demonstrated significant elevation in serum anti-MCV in RA patients versus controls which correlated with severity of inflammatory process as evidenced by the associated increase in the inflammatory biomarkers, and evidences of association of anti-mcv with higher incidence of radiographic progression in these patients (15-22), in contrast to this finding, Morbach et al. (22), found no significant difference.

The present study did not show the significant differences between sensitivity and specificity of ACCP and anti-MCV (sensitivity $85 \%, 81 \%$, specificity $96 \%$ and $95 \%$, respectively).

Liu et al., 2009 and Al-Shukaili et al.,2012 showed that the sensitivities of anti-MCV antibodies was the highest in comparison to anti-CCP antibodies and RF were $(78.2 \%$ and $72 \%),(61.8 \%$ and $52 \%)$, and $72.4 \%$ and $57 \%$ ), respectively. While a contradictory to Maraina et al., 2010, who stated that the sensitivity of RF was higher than the sensitivity of anti-CCP or anti-MCV antibodies. Also, contradictory to Bartoloni et al., 2012, who stated that, anti-MCV demonstrated lower sensitivity than antiCCP.

Roland et al., 2008, and Damjanovska et al 2009 , showed that, the specificity of antiMCV antibodies was the highest in comparison to anti-CCP antibodies.

While a contradictory to, Soos et al., 2007, Sghiri et al., 2008 and Al-Shukaili et al., 2012, who stated that; the specificity of antiCCP antibodies was higher than that of antiMCV antibodies. Positive anti MCV was also reported in SLE, SJogran syndrome, psoriatic arthritis.

In conclusion, our study suggests that ACCP is an informative diagnostic test for RA. anti-MCV have slight additional value. This statement is based on the somehow more sensitivity and specificity and the results of kappa, indicating positivity of ACCP in patients positive for anti-MCV and vice versa. 


\section{References}

1. Pedersen-Lane JH, Zurier RB, Lawrence DA (2007) Analysis of the thiol status of peripheral blood leukocytes in rheumatoid arthritis patients. J Leukoc Biol 81: 934-941

2. Scott DL, Wolfe F, Huizinga TWJ. Rheumatoid arthritis. Lancet 2010; 376: 1094-108.

3. Serre G (2001) Autoantibodies to filaggrin/deiminated fibrin (AFA) are useful for the diagnosis and prognosis of rheumatoid arthritis, and are probably involved in the pathophysiology of the disease. Joint Bone Spine 68: 103-105.

4. Aletaha DK, Neogi T, Silman AJ, et al. 2010 Rheumatoid arthritis classification criteria: an American College Of Rheumatology/European League against Rheumatism Collaborative initiative. Ann Rheum Dis 2010; 69: 1580-1588

5. Pruijn GJ, Wiik A, van Venrooij WJ. The Use of citrullinated peptides and proteins for the diagnosis of rheumatoid arthritis. Arthritis Res Ther 2010; 12: 203

6. Nicaise-Roland P, Nogueira L, Demattei $\mathrm{C}$, et al. Autoantibodies to citrullinated fibrinogen compared with anti-MCV and anti-CCP2 antibodies indiagnosing rheumatoid arthritis at an early stage: data from the French ESPOIR cohort. Ann Rheum Dis 2013; 72: 357-62

7. Nicaise-Roland P, Nogueira L, Demattei $\mathrm{C}$, et al. Autoantibodies to citrullinated fibrinogen compared with anti-MCV and anti-CCP2 antibodies indiagnosing rheumatoid arthritis at an early stage: data from the French ESPOIR cohort. Ann Rheum Dis 2013; 72: 357-62.

8. Levesque MC, Zhou Z, Moreland LW. Anti- Cyclic Citrullinated Peptide Testing for the diagnosing and Predictive Value Rheumatoid Arthritis and the Quest for Improved Sensitivity and Predictive Value. Arthritis Rheum 2009; 60: 2211-5.
9. Sghiri R, Bouajina E, Bargaoui D, et al. Value of anti-mutated citrullinated vimentin antibodies in diagnosing rheumatoid arthritis. Rheum atal Int 2008; 29: 59-62.

10. Mathsson L, Mullazehi M, Wick MC, et al. Antibodies against citrullinated vimentin in rheumatoid arthritis: higher sensitivity and extended prognostic vale concerning future radiographic progression as compared with antibodies against cyclic citrullinated peptides. Arthritis Rheum 2008; 58: 3645

11. Wagner E, Skoumal M, Bayerand PM, Klaushofer K. Antibody against mutated citrullinatedvimentin: A new sensitive marker in the diagnosis of rheumatoid arthritis. Rheumatol Int 2009; 29: 1315-21.

12. Mansour HE, Metwalt KM, Hassan IA, Elshamy HA, Elbeblawy MM. Antibody to mutated citrullinated vimentin in rheumatoid arthritis: diagnosticvalue, association with radiological damage and axial skeleton affection. Clin Med Insight Arthritis Musculoskelet Disorr 2010; 3: 33-42.

13. Innala L, Kokkonen H, Eriksson C, et al. Antibodies against mutated citrullinared vimentin are a better predictor of disease activity at 24 months in early rheumatoid arthritis than antibodies against cyclic citrullinated peptides. J Rheumatol 2008; 35: 1002-8.

14. Damjanovska L, Thabet MM, Levarth EW et al (2009): Diagnostic value of anti-MCV antibodies in differentiating early inflammatory arthritis. Ann Rheum Dis; 69:730_2.

15. Zhu T, Feng L (2013) Comparison of anti-mutated citrullinated vimentin, anti-cyclic citrullinated peptides, antiglucose-6-phosphate isomerase and anti-keratin antibodies and rheumatoid 
factor in the diagnosis of rheumatoid arthritis in Chinese patients. Int $\mathbf{J}$ Rheum Dis 16: 157-161.

16. Sariyildiz MA, Batmaz I, Guli Çetinçakmak M, Yıldız I, Nas K, et al. (2013) Relationship of the HLA-DRB1 alleles and seropositivity, anti-MCV, functional status and radiological damage in Turkish patients with rheumatoid arthritis. J Back Musculoskelet Rehabil 26: 63-70.

17. Mikuls TR, O’Dell JR, Stoner JA, Parrish LA, Arend WP, et al. (2004) Association of rheumatoid arthritis treatment response and disease duration with declines in serum levels of IgM rheumatoid factor and anti-cyclic citrullinated peptide antibody. Arthritis Rheum 50: 3776-3782.

18. Keskin G, Inal A, Keskin D, Pekel A, Baysal O, et al. (2008) Diagnostic utility of anti-cyclic citrullinated peptide and anti-modified citrullinated vimentin antibodies in rheumatoid arthritis. Protein Pept Lett 15: 314-317.
19. Zendman AJ, van Venrooij WJ, Pruijn GJ (2006) Use and significance of antiCCP autoantibodies in rheumatoid arthritis. Rheumatology (Oxford) 45: 20-25.

20. Liu X, Jia R, Zhao J, Li Z (2009) The role of anti-mutated citrullinated vimentin antibodies in the diagnosis of early rheumatoid arthritis. J Rheumatol 36;1136-1142.

21. Mathsson L, Mullazehi M, Wick MC, Sjöberg O, van Vollenhoven R, et al. (2008) Antibodies against citrullinated vimentin in rheumatoid arthritis: higher sensitivity and extended prognostic value concerning future radiographic progression as compared with antibodies against cyclic citrullinated peptides. Arthritis Rheum 58: 36-45.

22. Morbach H, Dannecker H, Kerkau T, Girschick HJ (2010) Prevalence of antibodies against mutated citrullinated vimentin and cyclic citrullinated peptide in children with juvenile idiopathic arthritis. Clin Exp Rheumatol 28: 800.

\section{How to cite this article:}

Saurabh Agarwal, Sippy Agrawal, Manoj Kumar and Arun Kumar Pandey. Sensitivity and specificity of Anti-mutated Citrullinated vimentin antibodies compared with Anti-cyclic Citrullinated Peptides in patients of Rheumatoid arthritis. Int.J.Curr.Microbiol.App.Sci. 5(2): 338-343. doi: http://dx.doi.org/10.20546/ijcmas.2016.502.039 